\title{
An unexpected case of bilateral kidneys studded with multiple abscesses, in a nondiabetic healthy woman - A case report
}

\author{
Vijaya Kamble', Kajal Mitra² \\ ${ }^{1}$ Associate Professor, Department of Radiodiagnosis, NKPSIMS and Research Centre, Nagpur, India-440019, ${ }^{2}$ Professor and Head of \\ Department, Department of Radiodiagnosis, NKPSIMS and Research Centre, Nagpur, India-440019
}

\section{A B S TR A C T}

\begin{abstract}
Bilateral multiple renal abscesses have been found in individuals with predisposing factors but are infrequently encountered in otherwise healthy individuals. In our case, a lady presented with history of fever and chills with bilateral renal angle tenderness. Ultrasonography and computed tomography showed both kidneys to be studded with multiple renal abscesses of varying sizes. USG Guided percutaneous aspiration was done to establish the correct diagnosis and identify the causative micro organism. Her blood culture and urine culture were negative. The patient was successfully treated with combined intravenous and oral course of antibiotics. In conclusion, as contrary to the usual belief, bilateral renal abscesses can occur in a healthy individual without any history of diabetes or immunocompromised status. In addition full recovery is possible solely with antibiotics.
\end{abstract}

Key words: Bilateral renal abscesses, Computed tomography, Healthy, Ultrasonography

\section{INTRODUCTION}

Bilateral multiple renal abscesses is a rare entity in itself and occurrence of it in a healthy individual is in again itself rare. This condition is usually associated with predisposing factors. The common predisposing conditions for renal abscesses described are systemic diseases such as diabetes mellitus, liver cirrhosis, underlying urinary obstructions, renal stones, renal or urologic disorders. ${ }^{1}$ The less common causes include intravenous drug abuse, chronic debilitating disease and immunocompromised status.

Early diagnosis and treatment of this entity is essential to reduce surgery related morbidity and mortality. Due to improved quality of imaging modalities like ultrasound and computed tomography, earlier diagnosis of renal abscesses is possible. Purpose of this case report was to document that bilateral multiple renal abscesses of these many numbers i.e about 12 to 15 , can occur in a healthy individual without any predisposing factor. Also early intervention in the form of USG guided percutaneous aspiration reveals precisely the causative micro-organism and full recovery can be achieved solely with antibiotics in the absence of positive urine and blood culture.

\section{CASE REPORT}

A 28 year old, young female, presented with history of high grade fever of six to eight days duration associated with chills. She also complained of dull aching pain in abdomen on and off since eight days. There was no history of pyuria, hematuria or burning micturition. She gave history of two episodes of vomiting about two days back. General physical examination demonstrated an elevated temperature of $101^{\circ} \mathrm{F}$ and tachycardia. Blood pressure and other vital signs were normal. On abdominal examination there was bilateral lumbar and renal angle tenderness. She was sent for ultrasound examination of abdomen. It revealed multiple, bilateral hypoechoic areas involving renal cortex and medulla in both kidneys. 
Right kidney was studded with approximately 12 to 15 hypoechoic areas and left kidney showed approximately 10 to 12 hypoechoic areas (Figure 1). These were ranging between 15 to $20 \mathrm{~mm}$ in size. Many of these hypoechoic areas were relatively small and were in the process of coalescing with each other. The largest hypoechoic area measured about $34 \times 24 \mathrm{~mm}$ in the inter polar region of left kidney. Few of these areas were showing low level internal echoes within. Both the kidneys were diffusely enlarged. Right kidney measured $11.1 \times 6.5 \mathrm{~cm}$ and left kidney measured $12.6 \times 7.2 \mathrm{~cm}$. Moderate free fluid was also noted in abdomen. Plain computed tomography of abdomen exhibited similar findings with bilateral, diffusely enlarged kidneys (Figure 2). Bilateral perinephric fat stranding was noted. There was no evidence of perinephric abscess. Contrast CT study was not done as the renal function was impaired raising serum creatinine and blood Urea levels (Table 1). However blood and urine cultures were negative. Also urine microscopy was negative for pus

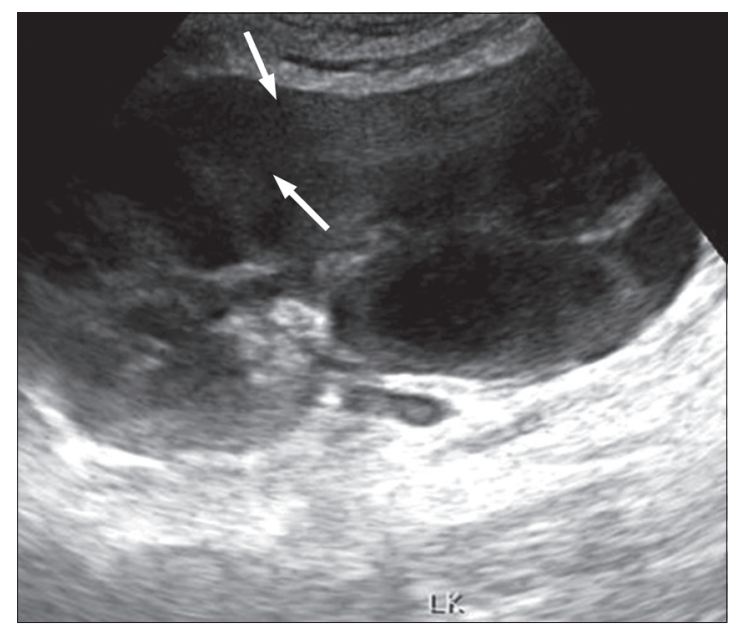

Figure 1: Ultrasonography shows multiple hypoechoic $(\rightarrow)$ lesions in the cortex of left kidney

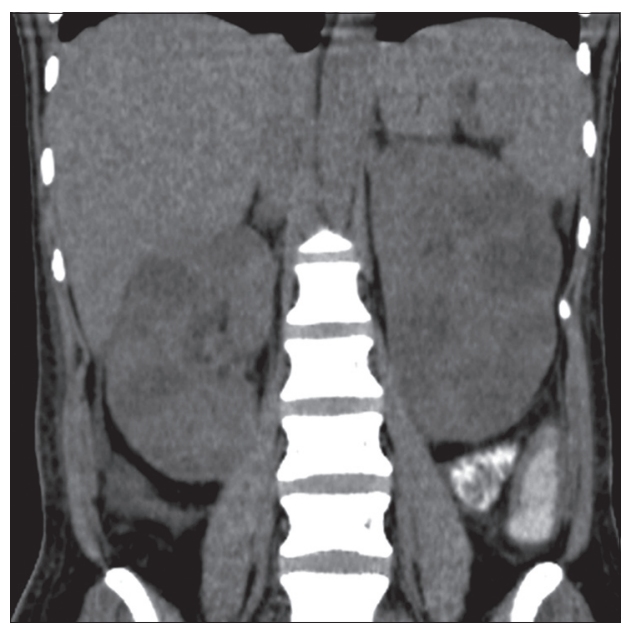

Figure 2: Plain coronal CT showing bilaterally enlarged kidneys and multiple bilateral hypodense areas in the renal parenchyma suggestive of abscesses cells. Her Total Leucocyte count was $10,400 \mathrm{~mm}^{3}$. Her Liver function tests were within normal range. Her fasting and post meal blood sugar level on admission was normal and remained within normal range in her admission period. Her peripheral smear showed normochromic and normocytic cells. ELISA test was negative which ruled out her immunocompromised status. Electrocardiogram was done which did not reveal any vegetation. On clinical examination and imaging studies, there was no evidence of any infective focus found in body. No obvious abscesses were noted in other solid organs. Based on the presumptive diagnosis of acute pyelonephritis with multiple bilateral renal abscesses (some of them in the process of evolution) and acute renal failure, USG Guided aspiration of the largest hypoechoic area from the left kidney was performed. About $10 \mathrm{cc}$ of pus was aspirated from the same and culture grew Escherichia coli, sensitive to ceftriaxone. Patient was started on injection Ceftazidime and Sulbactum $1.5 \mathrm{gm} \mathrm{BD}$ given intravenously, Injection Levoflox 500 OD given intravenously for 15 days followed by tablet Cetil $250 \mathrm{mg}$ BD and tablet Levoflox $500 \mathrm{mg}$ OD for next 15 days. Her fever was managed with paracetamol $500 \mathrm{mg}$ tablet SOS. Patient became afebrile within four days of starting the treatment. Her blood urea and serum creatinine levels came back to normal gradually in next 18 to 20 days. Patient was reviewed after three months, USG showed complete resolution of bilateral renal abscesses.

\section{DISCUSSION}

A renal abscess is defined as an abscess confined only to the renal parenchyma. ${ }^{1}$ It is known to develop from ascending infections of the lower urinary tract or by hematogenous seeding from primary infected sites. Ascending infections account for more than $75 \%$ of all renal abscesses and this usually involve medulla by gram negative organisms. ${ }^{1}$

In our case, there is no known predisposing condition. Bilateral renal abscesses in a young woman with type I diabetes is reported ${ }^{2}$ and is a known fact. However bilateral multiple renal abscesses in a healthy woman without any predisposing condition is relatively rare occurrence. Similar type of case is reported with no pre-existing systemic or renal disease in a male patient $t^{3}$ and in a previously healthy 11 year old girl. ${ }^{4}$

\begin{tabular}{lc} 
Table 1: Laboratory findings of the patient \\
\hline Hemoglobin & $7.7 \mathrm{~g} / \mathrm{dL}$ \\
Total leucocyte count & $10,400 \mathrm{~mm}^{3}$ \\
Serum creatine & $10 \mathrm{mg} / \mathrm{dL}$ (raised) \\
Blood urea & $260 \mathrm{mg} / \mathrm{dL}$ (raised) \\
Fasting blood sugar & $87 \mathrm{mg} / \mathrm{dL}$ (normal) \\
Post prandial blood sugar & $105 \mathrm{mg} / \mathrm{dL}$ (normal) \\
\hline
\end{tabular}


In this setting of no bacteriuria, i.e, negative urine culture and blood culture and also the patient did not give any history of lower urinary tract infection, patient presenting with multiple bilateral renal abscesses is quite rare. Usually patients with renal abscesses have positive urine culture. This clinical presentation of no positive history of UTI and laboratory investigations of negative urine culture and blood culture were misleading.

Renal abscess is divided into anatomic categoriesperinephric, renal cortical and corticomedullary. ${ }^{5}$ Renal abscess was also classified into three groups according to the size of abscess as follows: $<3 \mathrm{~cm}, 3-5 \mathrm{~cm}$ and $>5 \mathrm{~cm} .{ }^{1}$ In our case, one of the abscess in left kidney was of $>3 \mathrm{~cm}$ in size. Hence USG guided percutaneous drainage was performed. This resulted in collection of sample which was sent for culture sensitivity. Renal abscess is safely and effectively managed with proper intravenous and oral antibiotics.

Imaging wise both kidneys were studded with multiple renal abscesses about 12 to 15 in number in right kidney and about 10 to 12 in number in left kidney. In a study by Laufler, ${ }^{6}$ two hypoechoic areas were noted in right kidney and one hypoechoic area in left kidney. ${ }^{6}$ In another study by Bong Eun Lee et al $^{1}$ multiple abscesses (three or more) were found in nine patients, but they did not mention the exact number of abscesses on each side. As in our study, these many number of abscesses in both kidneys are not reported in the literature in a healthy individual which are conservatively managed.

\section{CONCLUSION}

In conclusion it can be said that multiple renal abscesses can occur in a healthy individual with this type of presentation, without any predisposing factors and negative blood and urine culture although it is rare. This should be kept in mind when evaluating a healthy individual with fever and lumbar pain. We recommend that the use of radiological interventions like percutaneous USG and CT guided drainage or aspiration contributes to accurate diagnosis and improved outcome of renal abscesses. These interventions help in the complete recovery of patient without the need of surgery and hence reduced mortality. This patient was successfully treated with a combined intravenous and oral course of antibiotics.

\section{REFERENCES}

1. Lee BE, Seol HY, Kim TK, Seong EY, Song SH, Lee DW, et al. Recent clinical overview of renal and perirenal abscesses in 56 consecutive cases. The Korean Journal of Internal Medicine 2008; 23:140-148.

2. Bevan JS, Griffiths GJ, Williams JD and Gibby OM. Bilateral renal cortical abscesses in a young woman with type 1 diabetes. Diabetic Medicine 1989; 6 (5): 454-457.

3. PR Chadwick and NB Thomas. Conservative management of a patient with bilateral renal abscesses. Journal of Infection 1994; 28 (1): 83-87.

4. Chaudhry $S$ and Bolt R. Bilateral renal abscess in a previously healthy 11-year-old girl. Eur J Pediatrics 2010; 169(11):1423-1425.

5. Anderson KA and McAninch JW. Renal abscess: Classification and review of 40 cases. Urology 1980; 16:333-338.

6. Laufler J, Grisaru-Soen G, Portnoy O and Mor Y. Bilateral renal abscesses in a healthy child. IMAJ 2002; 14(4):1150-1151. 